\title{
Scinax nebulosus (Spix, 1824) (Amphibia: Hylidae): review of distribution and new record from Sergipe, Brazil
}

\section{Eduardo José dos Reis Dias, Rony Peterson Santos Almeida, Maria Aldenise Xavier, Mayara de Lima Mota, Adriano da Cunha Lima and Igor Rios do Rosário}

Universidade Federal de Sergipe, Departamento de Biociências, Laboratório de Biologia e Ecologia de Vertebrados, Avenida Vereador Olímpio Grande s/n, Centro, CEP 49500-000, Itabaiana, SE, Brazil

* Corresponding author. E-mail: ejrdias@hotmail.com

\begin{abstract}
We present the first record of Scinax nebulosus for the State of Sergipe, in the Atlantic Forest, Brazil. Scinax nebulosus is a small hylid which inhabits the Amazon and Atlantic Forest. Its main microhabitat is temporary water bodies in tropical forests. This species can be recognized by the presence of many scattered glandules on the dorsal surface, especially on the head, upper eyelids and margins of the members. Some taxonomic studies and vocals records suggest careful evaluation of the taxonomic status of $S$. nebulosus along its geographical distribution.
\end{abstract}

Key words: Scinax, Atlantic Forest, State of Sergipe, Brazil

Scinax nebulosus (Spix, 1824) is a small-bodied hylid of the Amazon and Brazilian Atlantic Forest that ranges from southeastern Venezuela, the Guianas and Suriname, through the Amazon Basin of Brazil (Amapá, Amazonas, Rondônia, Acre, Pará, Tocatins) to northern Bolivia, part of Brazil Central (Mato Grosso, Mato Grosso do Sul and Goiás) and northeastern Brazil (Maranhão, Ceará, Rio Grande do Norte, Paraíba, Pernambuco, Alagoas and Bahia) (La Marca et al. 2004) (Table 1). This species was previously recognized as Hyla egleri Lutz, 1968 and was synonymised with $H$. nebulosa and designated the holotype of the first name as the neotype for Hyla nebulosa with type locality to Belém, State of Pará, Brazil (Hoogmoed and Gruber 1983). Later, Duellman and Wiens (1992) revised the taxonomy of some hylids and recognized the genus Scinax Wagler, 1830.

On 28 January 2013 during a nocturnal active search, we captured two specimens of Scinax nebulosus (18.52 and $23.38 \mathrm{~mm}$ snout vent length (SVL)) (Figure 1) at a fragment of Atlantic Forest in Capela municipality in Refúgio de Vida Silvestre Mata do Junco $\left(10^{\circ} 32^{\prime} 23^{\prime \prime} \mathrm{S}\right.$, $037^{\circ} \mathrm{O3}^{\prime} 43^{\prime \prime} \mathrm{W}$ ), State of Sergipe, Brazil (Figure 2)

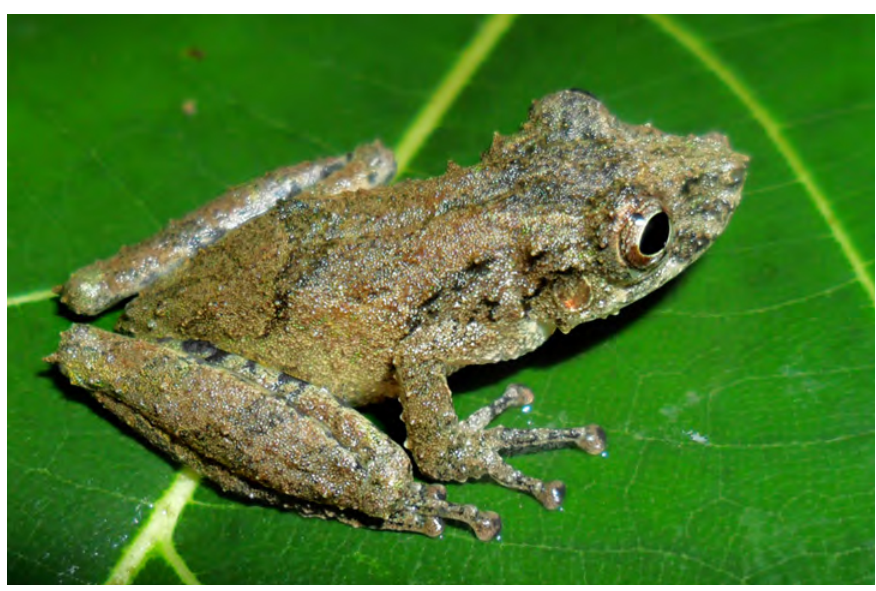

Figure 1. Live adult of Scinax nebulosus (Spix 1824) - LABEV A 859 (Photo by Rony P. S. Almeida).

(collection permit: 161/o4 - IBAMA/RAN). Specimens were housed in the amphibian collection of the Laboratório de Biologia e Ecologia de Vertebrados da Universidade Federal de Sergipe (LABEV A 859-860). Scinax nebulosus is a species without marked sexual characters, 22-23 $\mathrm{mm}$ snout to vent, can be recognized by the presence of many scattered glandules on the dorsal surface, especially on the head, upper eyelids, margins of the limbs, and sometimes on the edges of the spots, and interocular and sacral spots and dark dorsolateral areas are as inverted parentheses (Lutz 1973). The primary microhabitat of this species are temporary water bodies in tropical rainforest, also present in more open areas such as Cerrado moist savannah, as well as surviving to some extent in anthropogenic habitats, such as pastureland, secondary growth and rural gardens (La Marca et al. 2004). Comparisons of the vocals records of this species with others sympatric anurans of the same genus, suggest a need for careful evaluation of the taxonomic status of $S$. nebulosus along with its geographical distribution (Lima et al. 2004). Another study suggests that many or all specimens referred to 
Table 1. Localities of Scinax nebulosus (Spix, 1824) with respective coordinates and references. All Datum not mentioned in original references were treated as WGS84.

\begin{tabular}{|c|c|c|c|c|c|}
\hline $\begin{array}{l}\text { Brazilian state } \\
\text { (abbreviation) }\end{array}$ & Municipality & Collection site & Longitude & Latitude & $\begin{array}{l}\text { Reference or acronym of the scientific collec- } \\
\text { tion and specimen number }\end{array}$ \\
\hline SE & Capela & REVIS Mata do Junco & -37.0619 & $-010.5397^{*}$ & This research \\
\hline BA & Guaratinga & Fazenda Vista Bela & -39.7831 & $-016.5846^{* *}$ & Silvano and Pimenta 2003 \\
\hline BA & Canavieiras & Fazenda Monte Cristo & -38.9475 & $-015.6757^{* *}$ & Silvano and Pimenta 2003 \\
\hline$A C$ & Tarauacá & Floresta Estadual do Mogno & -71.5355 & $-007.9440^{*}$ & Pantoja and Fraga 2012 \\
\hline$A C$ & Tarauacá & Floresta Estadual Rio Gregório & -71.1769 & $-008.0679^{*}$ & Pantoja and Fraga 2012 \\
\hline$A L$ & Ibateguara & Usina Serra Grande - Coimbra & -35.8333 & $-008.9833^{*}$ & Carnaval 2002 \\
\hline$A L$ & Campo Alegre & Fazenda Pindoba & -36.3508 & $-009.7819^{* *}$ & UNESPRC/CFBH 16341-45, 16353-54 \\
\hline$A L$ & Passo de Camarajibe & not reported & -35.4933 & $-009.2383^{* *}$ & UNESPRC/CFBH 7541-47 \\
\hline AM & not reported & RDS Piagaçu-Purus & -61.6667 & $-004.0500^{*}$ & Waldez et al. 2013 \\
\hline AM & not reported & RDS Piagaçu-Purus & -63.5000 & $-005.4167^{*}$ & Waldez et al. 2013 \\
\hline AP & Macapá & Universidade Federal do Amapá & -51.0827 & $-000.0063^{*}$ & Pereira-Júnior et al. 2013 \\
\hline CE & Planalto de Ibiapaba & not reported & -41.1667 & $-005.0000^{*}$ & Loebmann and Haddad 2010 \\
\hline CE & Planalto de Ibiapaba & not reported & -40.7000 & $-003.3333^{*}$ & Loebmann and Haddad 2010 \\
\hline CE & Ubajara & Saída da cidade & -40.9211 & $-003.8544^{* *}$ & UNESPRC/CFBH 15859-61 \\
\hline GO & Quirinópolis & not reported & -50.4517 & $-018.4483^{* *}$ & UNESPRC/CFBH 4604 \\
\hline MA & not reported & Pátio da Ferrovia CVRD & -47.3736 & $-004.9506^{*}$ & Brasileiro et al. 2008 \\
\hline MA & not reported & Fazenda Três Lagoas & -47.1831 & $-004.7725^{*}$ & Brasileiro et al. 2008 \\
\hline MA & not reported & Fazenda Maravilha & -47.4286 & $-006.0106^{*}$ & Brasileiro et al. 2008 \\
\hline MA & not reported & Fazenda Jacuba & -47.3614 & $-006.2733^{*}$ & Brasileiro et al. 2008 \\
\hline MA & not reported & Fazenda Nova & -47.3642 & $-006.3017^{*}$ & Brasileiro et al. 2008 \\
\hline MA & not reported & Fazenda Ituaneira & -47.3928 & $-006.5303^{*}$ & Brasileiro et al. 2008 \\
\hline MA & Caxias & not reported & -43.3562 & $-004.8596^{* *}$ & Damacesno et al. 2012 \\
\hline MA & Pedrinhas & Parque Ambiental da Alumar & -42.7350 & $-003.7794^{* *}$ & Barreto et al. 2011 \\
\hline MA & Açailândia & Pátio Compahia Vale do Rio Doce & -47.5047 & $-004.9467^{* *}$ & UNESPRC/CFBH 15626-27 \\
\hline MA & Alcântara & not reported & -44.4147 & $-002.4089 * *$ & UNESPRC/CFBH 19171-075 \\
\hline MA & Balsas & not reported & -46.0356 & $-007.5325^{* *}$ & UNESPRC/CFBH 14012-013 \\
\hline MA & Estreito & Fazenda Ouro Verde & -47.4511 & $-06.5606^{* *}$ & UNESPRC/CFBH 18039 \\
\hline MA & Imperatriz & not reported & -47.4917 & $-005.5264^{* *}$ & UNESPRC/CFBH $8122-23$ \\
\hline MS & Corumbá & RPPN Acurizal & -56.7233 & $-018.7310^{*}$ & UFMT/UFMT-A 2493-94 \\
\hline MT & Nova Monte Verde & not reported & -57.6344 & $-010.4283^{*}$ & Ávila and Kawashita-Ribeiro 2011 \\
\hline MT & Nova Monte Verde & not reported & -57.6786 & $-010.3225^{*}$ & Ávila and Kawashita-Ribeiro 2011 \\
\hline MT & Tangará da Serra & not reported & -57.5608 & $-014.6331^{*}$ & Campos et al. 2013 \\
\hline MT & Alta Floresta & not reported & -56.0861 & $-009.8756^{* *}$ & UNESPRC/CFBH 21851 \\
\hline MT & Araputanga & PCH Ombreiras & -58.4572 & $-015.2558^{*}$ & $\begin{array}{l}\text { UFMT/UFMT-A 6977-078, 6981, 6983, 6987-88, } \\
\text { 7005-06, } 7011\end{array}$ \\
\hline MT & Aripuanã & Mineradora Geominas & -60.2631 & $-009.8603^{*}$ & UFMT/UFMT-A 7836, 7842, 7846 \\
\hline MT & Brasnorte & PCH Bocaiúva & -57.9950 & $-012.4251^{*}$ & UFMT/UFMT-A 10145-46 \\
\hline MT & Cáceres & ZPE de Cáceres & -57.8369 & $-016.5165^{*}$ & UFMT/UFMT-A 1081-82 \\
\hline MT & Juína & Diagem Mineração & -59.3230 & $-011.5308^{*}$ & UFMT/UFMT-A 6998, 7000-01 \\
\hline MT & Nova Bandeirantes & not reported & -57.8106 & $-009.8497^{* *}$ & UNESPRC/CFBH 21852 \\
\hline MT & Paranaíta & Margem do rio Teles Pires & -56.4767 & $-009.6647^{* *}$ & UNICAMP/ZUEC-AMP 16027, 16043-44 \\
\hline MT & Poconé & Morro do Carcará & -56.9485 & $-016.7845^{*}$ & UFMT/UFMT-A 7053 \\
\hline MT & São Félix do Araguaia & Alto da Boa Vista & -50.6692 & $-011.6172^{*}$ & UNICAMP/ZUEC-AMP 7490 \\
\hline MT & Tangará da Serra & Rio Sepotuba & -58.3104 & $-014.4346^{*}$ & UFMT/UFMT-A 2270, 2283 \\
\hline PA & Brasil Novo & $\begin{array}{l}\text { Private property next to } \\
\text { Transamazônica road }\end{array}$ & -52.5761 & $-003.3762^{*}$ & Oliveira et al. 2013 \\
\hline PA & Parauapebas & not reported & -49.9023 & $-006.0682^{* *}$ & Pinheiro et al. 2012 \\
\hline PA & Canaã dos Carajás & not reported & -49.8787 & $-006.4973^{* *}$ & Pinheiro et al. 2012 \\
\hline PA & Curionópolis & not reported & -49.5984 & $-006.1023^{* *}$ & Pinheiro et al. 2012 \\
\hline PA & Marabá & not reported & -49.1182 & $-005.3690^{* *}$ & Pinheiro et al. 2012 \\
\hline PA & São Félix do Xingu & not reported & -51.9951 & $-006.6451^{* *}$ & Pinheiro et al. 2012 \\
\hline PA & Altamira & Juruá & -52.2068 & $-003.2037^{* *}$ & Lima et al. 2004 \\
\hline PA & Belém & Museu Paraense Emílio Goeldi & -48.5045 & $-001.4562^{* *}$ & (Type locality) Hoogmoed and Gruber 1983 \\
\hline PA & Altamira & RESEX Riozinho do Anfrísio & -54.6551 & $-004.7594^{*}$ & Barros 2011 \\
\hline PA & Tucuruí & ZPVS do Lago de Tucuruí & -49.2000 & $-003.7167^{*}$ & Lima 2006 \\
\hline PA & Tucuruí & ZPVS do Lago de Tucuruí & -50.0000 & $-005.2500^{*}$ & Lima 2006 \\
\hline PA & Vitória do Xingú & not reported & -51.7519 & $-003.2391^{*}$ & Lima 2009 \\
\hline PA & Óbidos & Reserva Ecológica Grão Pará & -55.7285 & $-000.6303^{*}$ & Avila-Pires et al. 2010 \\
\hline PA & Altamira & Acesso ao Acampamento Juruá & -52.2064 & $-003.2033^{*}$ & UNICAMP/ZUEC-AMP 7240, 7253-54, 7331, 7395 \\
\hline
\end{tabular}


Table 1. Continued.

\begin{tabular}{|c|c|c|c|c|c|}
\hline $\begin{array}{l}\text { Brazilian state } \\
\text { (abbreviation) }\end{array}$ & Municipality & Collection site & Longitude & Latitude & $\begin{array}{l}\text { Reference or acronym of the scientific } \\
\text { collection and specimen number }\end{array}$ \\
\hline PA & Bragança & not reported & -46.7492 & $-001.0483^{*}$ & PUCRS/MCP-ANFIBIOS 12164, 12189 \\
\hline PA & Itaituba & Jardim do Ouro & -55.8953 & $-006.2656^{*}$ & UFES/UFES-CTA 1991, 1992 \\
\hline PA & Oriximiná & Laudicas & -55.8661 & $-001.7655^{* *}$ & UNICAMP/ZUEC-AMP 17645-47 \\
\hline PB & João Pessoa & APP Mata do Buraquinho & -34.8650 & $-007.1450^{*}$ & Santana et al. 2008 \\
\hline PB & Mamanguape & not reported & -35.1261 & $-6.8393^{* *}$ & Lutz 1973 \\
\hline PE & $\begin{array}{l}\text { Cabo de Santo } \\
\text { Agostinho }\end{array}$ & Compesa-Gurjau & -35.0333 & $-008.2333^{*}$ & Carnaval 2002 \\
\hline PE & Caruaru & Brejo dos Cavalos & -36.0167 & $-008.3667^{*}$ & Carnaval 2002 \\
\hline PE & Jaqueira & Usina Frei Caneca & -35.8333 & $-008.7167^{*}$ & Carnaval 2002 \\
\hline PE & Timbaúba & Usina Cruangui, Água Azul & -35.3667 & $-007.6000^{*}$ & Carnaval 2002 \\
\hline PE & Brejo Madre de Deus & Sítio Bituri & -36.4000 & $-008.2000^{*}$ & Carnaval 2002 \\
\hline PI & Parnaíba & not reported & -41.7770 & $-002.9051^{* *}$ & Loebmann and Mai 2008 \\
\hline PI & Luiz Correia & not reported & -41.6658 & $-002.8836^{* *}$ & Loebmann and Mai 2008 \\
\hline RN & Macaíba & Escola Agrícola de Jundiaí & -35.3670 & $-005.8852^{*}$ & Magalhães et al. 2013 \\
\hline RO & Jaraú & not reported & -62.4852 & $-010.4043^{*}$ & Piatti et al. 2012 \\
\hline RO & Urupá & Sítio Boa Vontade & -62.2133 & $-011.0714^{*}$ & Silva and Silva 2010 \\
\hline RO & Espigão do Oeste & Fazenda Jaburi & -60.6833 & $-011.5833^{*}$ & Bernarde 2007 \\
\hline RO & Espigão do Oeste & Fazenda Jaburi & -60.7500 & $-011.6333^{*}$ & Bernarde 2007 \\
\hline RO & Alto Alegre dos Parecis & Fazenda Santa Rita & -61.8506 & $-012.1281^{*}$ & UFMT/UFMT-A 7811, 7813 \\
\hline RO & Espigão D'Oeste & Fazenda Jaburi & -61.0128 & $-011.5247^{* *}$ & UNESPRC/CFBH 5111-012 \\
\hline TO & Jalapão & not reported & -46.7500 & $-010.5503^{* *}$ & Sturaro et al. 2010 \\
\hline TO & Araguaína & Localidade P35 & -48.2072 & $-007.1911^{* *}$ & UNESPRC/CFBH 11434 \\
\hline TO & Lizarda & Balneário & -46.6731 & $-009.5942^{* *}$ & UNESPRC/CFBH 13233 \\
\hline TO & Porto Nacional & Estrada da Fazenda Lazer & -48.4172 & $-010.7081^{* *}$ & UNESPRC/CFBH 16496-97 \\
\hline TO & São Félix do Tocantins & Fazenda Barrinha & -46.6594 & $-010.1683^{* *}$ & UNESPRC/CFBH 13245 \\
\hline
\end{tabular}

*Exact geographical position; ** Not exact geographical position; Reserva Extrativista (RESEX); Refúgio de Vida Silvestre (REVIS); Área de Proteção Permanente (APP); Zonas de Preservação de Vida Silvestre (ZPVS); Universidade Estadual Paulista de Rio Claro (UNESPRC); Coleção de Anfíbios da UNESP, Celio Fenando Baptista Haddad (CFBH); Universidade Federal de Mato Grosso (UFMT); Universidade Federal do Espírito Santo (UFES); Universidade Estadual de Campinas (UNICAMP); Pontifícia Universidade Católica do Rio Grande do Sul (PUCRS).

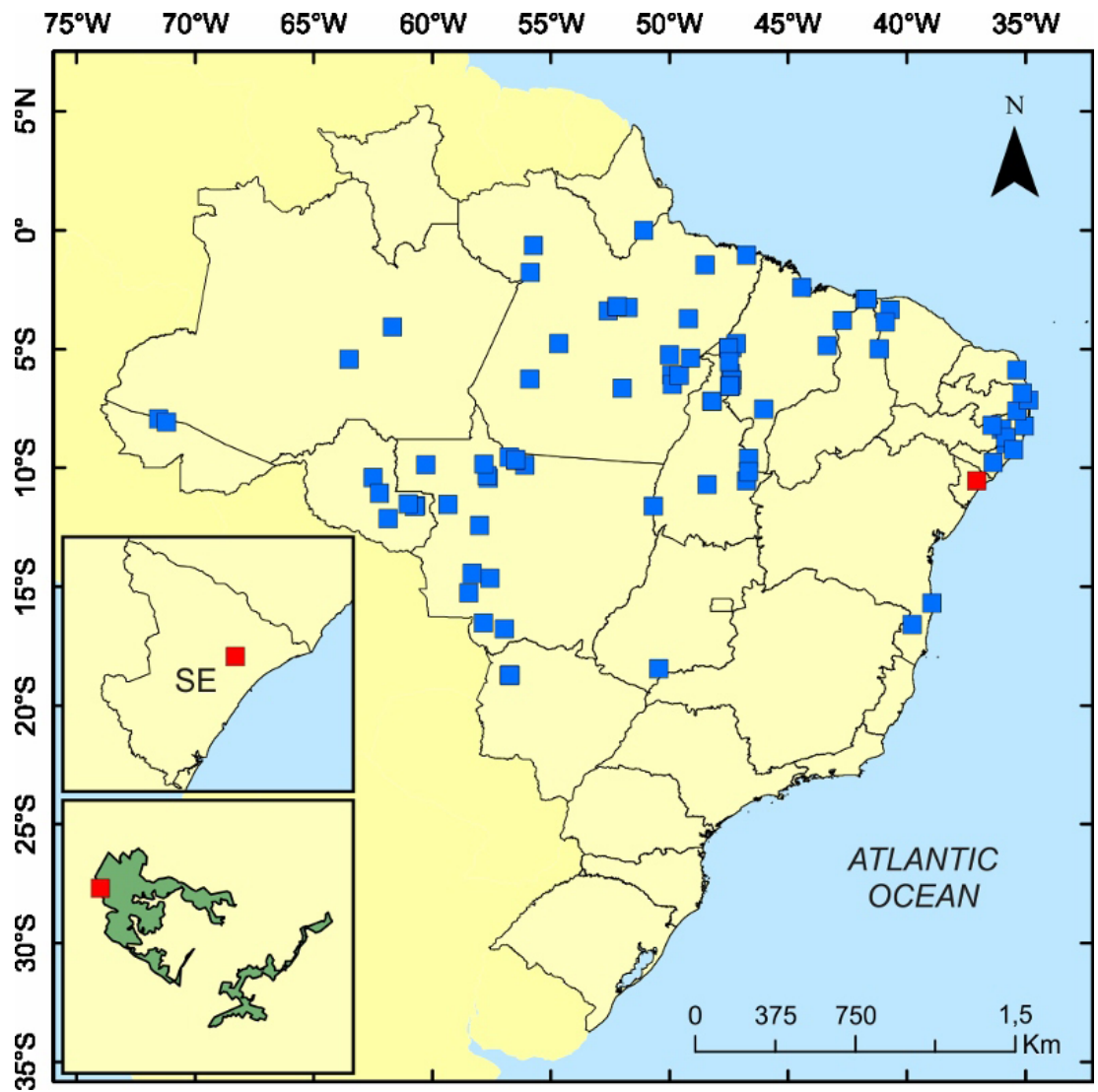

Figure 2. Known records of Scinax nebulosus, Brazil. BA: Bahia; SE: Sergipe; AL: Alagoas; PE: Pernambuco; PB: Paraíba; RN: Rio Grande do Norte; CE: Ceará; MA; Maranhão; PA: Pará; AP: Amapá; AM: Amazonas; RO: Rondônia; AC: Acre, MT: Mato Grosso; MS: Mato Grosso do Sul; GO: Goiás and TO: Tocantins. Blue square represent previously reported localities and red square represents the new record in Refugio de Vida Silvestre Mata do Junco, SE (map in green). 
S. nebulosus from central and western Brazil and Bolivia in fact are S. pedromedinae (Hoogmoed and Avila-Pires 2011) and Lutz (1973) also suggested differences between the forms of the north and the northeastern. Thus, due these taxonomic uncertainties we also believe that the different populations throughout the geographic distribution (shown in this paper) can possibly be different taxonomic entities.

\section{ACKNOWLEDGEMENTS}

We thank G.J.B Moura for confirming the identity of the species. R.J. Oliveira for help in collecting anurans. M. Tsuchiya for critically reviewing. The Secretaria de Meio Ambiente e Recursos Hídricos of Sergipe state for logistic support and collection permits. The Instituto Brasileiro do Meio Ambiente e Recursos Naturais Renováveis - IBAMA conceded the collection permits. The Cordenação de Pesquisas (COPES) of the Universidade Federal de Sergipe provided a graduate fellowship.

\section{LITERATURE CITED}

Avila-Pires, T.C.S., M.S. Hoogmoed and W.A. da Rocha. 2010. Notes on the Vertebrates of northern Pará, Brazil: a forgotten part of the Guianan Region, I. Herpetofauna. Boletim do Museu Paraense Emílio Goeldi Ciências Naturais 5(1): 13-112. http:// scielo.iec.pa.gov.br/pdf/bmpegcn/v6n1/v6n1ao3.pdf

Ávila, R.W. and R.A. Kawashita-Ribeiro. 2011. Herpetofauna of São João da Barra Hydroelectric Plant, state of Mato Grosso, Brazil. Check List 7(6): 750-755. doi: 10.15560/11014

Barreto, L., L.E.S. Ribeiro and M.C. Nascimento. 2011. Caracterização da herpetofauna em áreas da Amazônia do Maranhão; 203218, in: M.B. Martins and T.G. de Oliveira (ed.). Amazônia Maranhense: diversidade e conservação. Belém: Museu Paraense Emílio Goeldi. http://ppbio.museu-goeldi.br/sites/default/files/ Meu_livro.pdf

Barros, F.B. 2011. Biodiversidade, uso de recursos naturais e etnoconservação na Reserva Extrativista Riozinho do Anfrísio (Amazônia, Brasil). Tese de Doutorado. Lisboa: Universidade de Lisboa. 223 pp.

Bernarde, P.S. 2007. Ambientes e temporada de vocalização da anurofauna no Município de Espigão do Oeste, Rondônia, Sudoeste da Amazônia- Brasil (Amphibia: Anura). Biota Neotropica 7(2): 87-92. http://www.scielo.br/pdf/bn/v7n2/a1ovo7n2.pdf

Brasileiro, C.A., E.M. Lucas, H.M. Oyamaguchi, M.T.C. Thomé and M. Dixo. 2008. Anurans, Northern Tocantins River Basin, states of Tocantins and Maranhão, Brazil. Check List 4(2): 185-197. doi: http://www.checklist.org.br/getpdf?SLoo1-o8

Campos, V.A., F.H. Oda, L. Juen, A. Barth and A. Dartora. 2013 Composição e riqueza de espécies de anfíbios anuros em três diferentes habitat em um agrossistema no Cerrado do Brasil central. Biota Neotropica 13(1): 124-132. http://www.scielo.br/ $\mathrm{pdf} / \mathrm{bn} / \mathrm{v} 13 \mathrm{n} 1 / 14 . \mathrm{pdf}$

Carnaval, A.C.O.Q. 2002. Phylogeography of four frog species in forest fragments of northeastern Brazil - a preliminary study. Integrative and Comparative Biology 42(5): 913-921. doi: 10.1093/ $\mathrm{icb} / 42.5 .913$

Damasceno, E.C.M.S., K.C. Bezerra, E. Fraga and M.C. Barros. 2012. Molecular characterization of Scinax nebulosus (Spix, 1824) (Anura, Hylidae) of occurance in the cerrado of Maranhão. In $58^{\circ}$ Congresso Brasileiro de Genética. Paraná: Rafain Palace Hotel e Convention Center.
Duellman, W.E. and J.J. Wiens. 1992. The Status of the hylid frong genus Ololygon and the recognition of Scinax Wagler, 1830. Occasional Papers of the Museum of Natural History, University of Kansas 151: 1-23. doi: http://biostor.org/reference/573

Hoogmoed, M.S. and T.C.S. Avila-Pires. 2011. On the presence of Scinax pedromedinae (Henle, 1991) (Amphibia: Anura: Hylidae) in Amazonian Brazil and northern Peru. Boletim do Museu Paraense Emílio Goeldi Ciências Naturais 6(3): 263-271. http:// www.museu-goeldi.br/editora/bn/artigos/cnv6n3_2011/ presence\%28hoogmoed\%29.pdf

Hoogmoed, M.S. and U. Gruber. 1983. Spix and Wagler type specimens of reptiles and amphibians in the Natural History Musea in Munich (Germany) and Leiden (The Netherlands). Spixiana Supplement 9: 319-415. doi: http://biostor.org/reference/109582

La Marca, E., R. Reynolds and C. Azevedo-Ramos. 2004. Scinax nebulosus; in: IUCN. 2013. IUCN Red List of threatened species. Version 2013.2. Accessed at http://www.iucnredlist.org, 6 February 2014.

Lima, L.P., R.P. Bastos and A.A. Giaretta. 2004. A new Scinax Wagler, 1830 of the $S$. rostratus group from Central Brazil (Amphibia, Anura, Hylidae). Arquivos do Museu Nacional 62(4): 505-512. http://www.publicacao.museunacional.ufrj.br/Arquivos/Arq 622004/Arq624/6Arq624.pdf

Lima, J.F.R. 2006. Composição e riqueza de espécies de anuros (Amphibia) em fragmentos florestais no Lago de Tucuruí, Pará. Dissertação de Mestrado. Belém: Universidade Federal do Pará. $48 \mathrm{pp}$.

Lima, A.A. 2009. Composição, riqueza e abundância de espécies de anfíbios na região do médio Rio Xingu. Dissertação de Mestrado. Belém: Universidade Federal do Pará. 89 pp.

Loebmann, D. and A.C.G. Mai. 2008. Amphibia, Anura, coastal zone, state of Piauí, northeastern Brazil. Check List 4(2): 161-170. doi: http://www.checklist.org.br/getpdf?SLoo4-o8

Loebmann, D. and C.F.B. Haddad. 2010. Amphibians and reptiles from a highly diverse area of the Caatinga domain: composition and conservation implications. Biota Neotropica 10(3): 227-256. doi: 10.1590/S1676-06032010000300026

Lutz, B. 1973. Brazilian species of Hyla. Austin: University of Texas Press. $260 \mathrm{pp}$

Magalhães, F.M., A.K.B.P. Dantas, M.R.M. Brito, P.H.S. Medeiros, A.F. Oliveira, T.C.S.O. Pereira, M.H.C. Queiroz, D.J. Santana, W.P. Silva and A.A. Garda. 2013. Anurans from an Atlantic Forest-Caatinga ecotone in Rio Grande do Norte State, Brazil. Herpetology Notes 6: 1-10. http://www.herpetologynotes.seh-herpetology. org/Volume6_PDFs/Magalhaes_Herpetology_Notes_Volume6_ page1-10.pdf

Oliveira, E.A., E.J.H. Ruz and F.B. Barros. 2013. Herpetofauna delas Proximidades de la Caverna Planaltina, Brasil Novo, Pará (Amazonia Brasileña). Herpetotropicos 9(1-2): 55-68. http://150.185.138.105/ ojs/index.php/herpetotropicos/article/view/4487/4278

Pantoja, D.L. and R. de Fraga. 2012. Herpetofauna of the Reserva Extrativista do Rio Gregório, Juruá Basin, southwest Amazonia, Brazil. Check List 8(3): 360-374. doi: http://www.checklist.org. $\mathrm{br} /$ getpdf?SLo91-11

Pereira-Júnior, A.P., C.E.C. Campos and A.S. Araújo. 2013. Composição e diversidade de anfíbios anuros do campus da Universidade Federal do Amapá. Biota Amazônia 3(1): 13-21. http://periodicos. unifap.br/index.php/biota/article/view/589/pdf_6o

Piatti, L., P.M.O. Amaro, J.F.J. Araújo, V.Q.A. Sanches and P.S. Bernarde. 2012. Anurans of a disturbed area in Jarú, Rondônia, Brazil. Check List 8(1): 83-87. doi: http://www.checklist.org.br/ getpdf?SLo49-11

Pinheiro, L.C., Y.O.C. Bitar, U. Galatti, S. Neckel-Oliveira and M.C. Santos-Costa. 2012. Amphibians from southeastern state of Pará: Carajás Region, northern Brazil. Check List 8(4): 693-702. doi: http://www.checklist.org.br/getpdf?SLo14-12 
Santana, G.G., W.L.S. Vieira, G.A. Pereira-Filho, F.R. Delfim, Y.C.C. Lima and K.S. Vieira. 2008. Herpetofauna em um fragmento de Floresta Atlântica no Estado da Paraíba, Região Nordeste do Brasil. Biotemas 21(1): 75-84. doi: 10.5007/2175-7925.2008v21n1p75

Silva, F.C. and M.O. Silva. 2010. Distribuição espacial e temporal de anuros em dois ambientes: floresta ciliar e pastagem no Município de Urupá, Rondônia. Revista Científica da Faculdade de Educação e Meio Ambiente 1(1): 65-83. doi: http://www.faema.edu.br/ revistas/index.php/Revista-FAEMA/article/view/15/9

Silvano, D.L. and B.V.S. Pimenta. 2003. Diversidade e distribuição de anfíbios na Mata Atlântica do sul da Bahia. In Corredor de biodiversidade da Mata Atlântica do sul da Bahia (P.I. Prado, E.C. Landau, R.T. Moura, L.P.S. Pinto, G.A.B. Fonseca \& K. Anger, eds). IESB; CI; CABS; UFMG; UNICAMP, Ilhéus.

Sturaro, M.J., J.F.M. Sarmento, A.A. Lima, H.M. Chalkidis and R.A.T. Rocha. 2010. New records and distribution of the treefrog
Scinax rostratus (Peters, 1863) (Amphibia: Anura: Hylidae). Herpetology Notes 3: 161-166. http://www.herpetologynotes. seh-herpetology.org/Volume3_PDFs/Sturaro_et_al_ Herpetology_Notes_Volume3_pages161-166.pdf

Waldez, F., M. Menin and R.C. Vogt. 2013. Diversidade de anfíbios e répteis Squamata na região do baixo rio Purus, Amazônia Central, Brasil. Biota Neotropica 13(1): 299-316. doi 10.159o/ S1676-06032013000100029

Authors' contribution statement: All authors worked on data collection and preparation of this work.

Received: January 2015

Accepted: April 2015

Academic editor: Camila C. Both 